\title{
SUBSTITUIÇÃO DE MOTOR PADRÃO POR MOTOR DE ALTO RENDIMENTO COM INVERSOR DE FREQUÊNCIA EM TORRE DE RESFRIAMENTO*
}

Leandro Mendes Pinto ${ }^{1}$ Pedro Magalhães Sobrinho ${ }^{2}$

\section{Resumo}

Este artigo apresenta um estudo de analise da viabilidade da substituição de 1 motor padrão utilizado em uma torre de resfriamento por 1 motor de alto rendimento Premium acionado por um Inversor de frequência interligado ao controlador de temperatura. Nas Torres de Resfriamento, ao implantar uma solução que pode a variar a velocidade dos ventiladores de acordo com a temperatura ambiente e a exigência de resfriamento do processo, pode-se obter grandes ganhos em economia de energia. Neste cenário, os motores elétricos têm papel determinante pelo amplo uso na indústria devido a sua versatilidade e eficácia. Por isso, melhorias e inovações são sempre necessárias para otimizar processos e aumentar a lucratividade

Palavras-chave: Motores; Eficiência energética; Torre de resfriamento.

\section{STANDARD MOTOR REPLACEMENT FOR HIGH PERFORMANCE MOTOR WITH FREQUENCY INVERTER IN COOLING TOWER}

\section{Abstract}

This article presents a study of 1 standard engine replacing the feasibility of analysis used in a cooling tower for 1 high-efficiency motor Premium driven by a frequency inverter connected to the temperature controller. In Cooling Towers, to deploy a solution that can vary the speed of the fans according to the ambient temperature and the cooling requirement of the process, one can get great gains in energy efficiency. In this scenario, the electric motors have decisive role for widespread use in the industry due to its versatility and effectiveness. Therefore, improvements and innovations are always needed to optimize processes and increase profitability.

Keywords: Engines; Energy efficiency; Cooling tower.

1 Engenharia Elétrica e Eletrônica, Engenheiro de Manutenção, GERDAU, Pindamonhangaba, São Paulo, Brasil.

2 Mestrando Transmissão e conversão de energia, Engenheiro Eletricista, Energia, UNESP, Guaratinguetá, São Paulo, Brasil. 


\section{INTRODUÇÃO}

O setor industrial responde por $35,8 \%$ de toda energia consumida no Brasil [1]. No entanto, não existe uma política governamental de longo prazo específica para o uso eficiente da energia na indústria. Isso se reflete na baixa prioridade dos programas federais de eficiência energética, nos investimentos de fundos setoriais de eficiência energética e nas condições de financiamento.

Segundo Ministério do Meio Ambiente [2]. Por definição a eficiência energética consiste da relação entre a quantidade de energia empregada em uma atividade e aquela disponibilizada para sua realização, a promoção da eficiência energética abrange a otimização das transformações, do transporte e do uso dos recursos energéticos, desde suas fontes primárias até seu aproveitamento. Adotam-se, como pressupostos básicos, a manutenção das condições de conforto, de segurança e de produtividade dos usuários, contribuindo, adicionalmente, para a melhoria da qualidade dos serviços de energia e para a mitigação dos impactos ambientais.

Os sistemas motrizes têm particular importância devido à sua grande participação no consumo de eletricidade. É muito comum encontrarem-se motores superdimensionados em operação, o que gera significativo desperdício de energia elétrica e também o uso inadequado dos motores é fator de desperdício, pois muitas vezes operam em vazio (sem carga). Assim, ao dimensionar e utilizar os sistemas de força motriz de forma adequada, o impacto nos custos operacionais e no meio ambiente pode ser reduzido significativamente [3].

Atualmente nas Indústrias, os custos operacionais representam os principais gastos. Estes podem ser divididos em custos de manutenção e custos de energia. Os gastos com energia são os mais significativos nos custos de produção.

Os elevados custos de energia enfrentados pela indústria no país, assim como a necessidade constante de atualização e melhoria dos equipamentos para se obter ganhos em produtividade, exigem que as empresas façam análises rotineiras de seu parque. Neste contexto, analisando a característica de operação das torres de resfriamento de uma grande empresa siderúrgica identificou-se um potencial de economia, pois a operação é variável e uma adequação da instalação respeitando estas variações pode-se obter ganhos.

A Torre de Resfriamento é um equipamento para resfriamento da água usada em processos diversos, seja para sistemas de ar condicionado ou refrigeração de equipamentos.

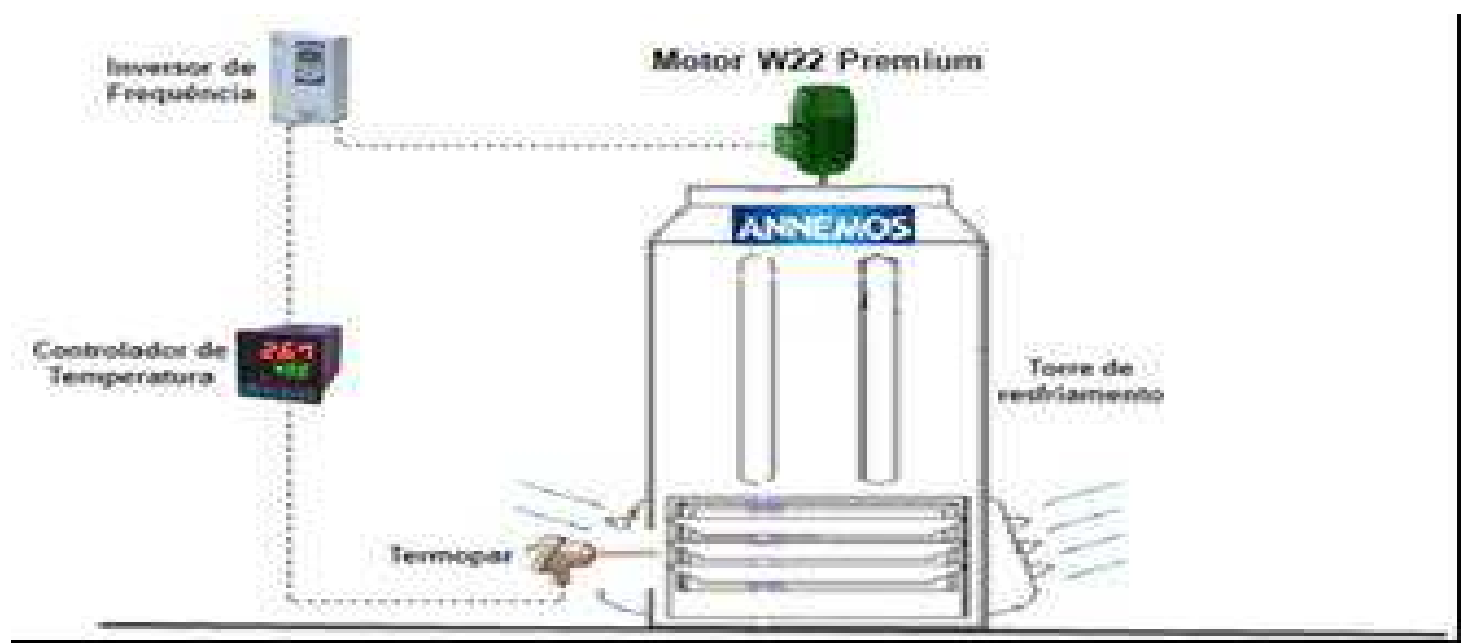

Fig. 1. Exemplo de Torres de Resfriamento [4]. 
Torres de resfriamento de água são importantes equipamentos ligados diretamente aos processos produtivos dos mais variados ramos das indústrias. Importantes avanços na área de pesquisa têm contribuído para o desenvolvimento de modelos matemáticos cada vez mais robustos os quais objetivam representar os fenômenos físicos no interior da torre, como também na otimização de projetos e diagnósticos de desempenho das torres de resfriamento de água. Em várias partes do mundo estudos vêm sendo conduzidos abordando de alguma forma o assunto torres de resfriamento, isto se deve em razão a sua importância associada a fatores de redução de custos operacionais e a ligação forte com o Meio ambiente, no quesito recirculação de água.

Para tanto neste estudo será mostrado uma torre utilizada para resfriamento de água de retorno de um forno elétrico de Aciaria de uma grande indústria siderúrgica.

Durante o ano, o valor médio da temperatura pode ser menor que a considerada devido às condições climáticas local. Variações de processo devido sazonalidades de produção e diferenças de temperatura durante o tempo de operação (turnos diurno e noturno) também podem fazer com que a temperatura média real fique abaixo da estimada no projeto.

Tais variáveis podem fazer com que a torre de resfriamento opere "sobredimensionada", resfriando a água mais do que o processo solicita e, portanto consumindo energia elétrica de forma desnecessária. Este cenário pode ser mudado com o controle da temperatura da água através de um sensor e um controlador, cujo sinal é transmitido ao inversor de frequência, que varia a rotação do motor elétrico do ventilador, insuflando mais ou menos ar.

\section{MATERIAIS E MÉTODOS}

\subsection{Metodologia}

Uma produção variável, ou a operação no inverno versus o verão, exigem capacidades distintas de resfriamento que são automaticamente ajustadas. Estas variações são reconhecidas pelo controlador de temperatura, que por sua vez envia a informação ao inversor de frequência o qual altera a velocidade do motor do ventilador, de acordo com a condição de operação, proporcionando uma substancial economia de energia, pois o motor elétrico utiliza somente a energia necessária para o trabalho solicitado, sem desperdícios.

Para obtenção dos resultados será monitorado o consumo de energia elétrica do motor Standard do ventilador da torre, operando sem nenhum controle de velocidade e após será realizado a aplicação da solução de eficiência energética substituindo o motor Standard pelo motor W22 Premium + Inversor de Frequência, e será realizado assim um comparativo real de consumo entre os motores. A solução de eficiência energética será aplicada, mantendo a igualdade do processo, tempo de operação, potência dos motores, especificação dos ventiladores, enfim, todas as características em geral. 


\subsection{Levantamentos de Dados}

Tabela 1 Dados do Motor Atual instalado

\begin{tabular}{|c|c|}
\hline \multicolumn{2}{|c|}{ Dados do Motor Elétrico Atual } \\
\hline Marca & Búfalo \\
\hline Carcaça & F-28OM \\
\hline Modelo & Horizontal \\
\hline Volt/Amper & $440 \mathrm{~V} / 177 \mathrm{~A}$ \\
\hline Frequência & $60 \mathrm{~Hz}$ \\
\hline Categoria & $\mathrm{B}$ \\
\hline Rotação & $1770 \mathrm{rpm}$ \\
\hline Nosérie & $\mathrm{A5761.1}$ \\
\hline Potência & $150 \mathrm{CV}$ \\
\hline F.S & 1,25 \\
\hline Isolação & $\mathrm{F}$ \\
\hline
\end{tabular}

Tabela 2 Dados do motor elétrico para substituição

\begin{tabular}{|c|c|}
\hline Dados do Motor Elétrico para Substituir \\
\hline Marca & WEG \\
\hline Carcaça & $280 S / M$ \\
\hline Modelo & Horizontal \\
\hline Volt/Amper & $440 V / 150 A$ \\
\hline Frequência & $60 H z$ \\
\hline Categoria & B \\
\hline Rotação & 1770 rpm \\
\hline No série & A5761.1 \\
\hline Potência & $125 \mathrm{CV}$ \\
\hline Reg Se & $\mathrm{F}$ \\
\hline Isolação & \\
\hline \multicolumn{2}{|c|}{ Dados do inversor a ser instalado } \\
\hline Inversor de Frequência-Trifásico-380-480V-180A CFW11 0180T4SZ \\
\hline
\end{tabular}

Levantamento dos Custos aproximados

Custo do motor + Inversor

Motor alto rendimento 125cv 4P 280S/M W22 - WEG

Aproximadamente $\mathbf{R} \mathbf{\$ 2 0 . 8 6 1 , 2 0}$

Inversor de Frequência - Trifásico CFW11 0180T4SZ.

\section{Aproximadamente $\mathbf{R} \mathbf{\$ 1 5 . 7 6 8 , 3 5}$}

Tabela 3 Valores medidos durante $24 \mathrm{hs}$ de trabalho

\begin{tabular}{|l|l|r|r|r|r|}
\hline Data & Hora & Tensão (V) & Corrente (A) & Potência (VA) & Fator de Potência \\
\hline $30 / 10 / 2014$ & $10: 04: 46778 \mathrm{~ms}$ & 436,44 & 82,2 & 56746,672 & 0,88 \\
\hline $30 / 10 / 2014$ & $11: 04: 46778 \mathrm{~ms}$ & 442,4 & 81,9 & 56933,34 & 0,88 \\
\hline $30 / 10 / 2014$ & $12: 04: 46778 \mathrm{~ms}$ & 445,56 & 81,4 & 57200,008 & 0,88 \\
\hline $30 / 10 / 2014$ & $13: 04: 46778 \mathrm{~ms}$ & 441,28 & 81,9 & 57186,672 & 0,88 \\
\hline $30 / 10 / 2014$ & $14: 04: 46778 \mathrm{~ms}$ & 442,5 & 81,8 & 56840,008 & 0,88 \\
\hline $30 / 10 / 2014$ & $15: 04: 46778 \mathrm{~ms}$ & 443,58 & 82,4 & 57546,672 & 0,88 \\
\hline $30 / 10 / 2014$ & $16: 04: 46778 \mathrm{~ms}$ & 439,9 & 82,6 & 57306,672 & 0,88 \\
\hline $30 / 10 / 2014$ & $17: 04: 46778 \mathrm{~ms}$ & 446,72 & 81,6 & 57093,34 & 0,87 \\
\hline $30 / 10 / 2014$ & $18: 04: 46778 \mathrm{~ms}$ & 436,88 & 82,8 & 57173,34 & 0,88 \\
\hline $30 / 10 / 2014$ & $19: 04: 46778 \mathrm{~ms}$ & 437,24 & 82,3 & 57360,008 & 0,88 \\
\hline $30 / 10 / 2014$ & $20: 04: 46778 \mathrm{~ms}$ & 442,12 & 82,4 & 57453,34 & 0,88 \\
\hline $30 / 10 / 2014$ & $21: 04: 46778 \mathrm{~ms}$ & 435,66 & 83,6 & 57466,672 & 0,88 \\
\hline $30 / 10 / 2014$ & $22: 04: 46778 \mathrm{~ms}$ & 434,02 & 82,8 & 57026,672 & 0,88 \\
\hline $30 / 10 / 2014$ & $23: 04: 46778 \mathrm{~ms}$ & 436,68 & 82,5 & 57080,008 & 0,88 \\
\hline $31 / 10 / 2014$ & $00: 04: 46778 \mathrm{~ms}$ & 432,04 & 83,4 & 56920,008 & 0,88 \\
\hline $31 / 10 / 2014$ & $01: 04: 46778 \mathrm{~ms}$ & 442,1 & 82 & 56946,672 & 0,88 \\
\hline $31 / 10 / 2014$ & $02: 04: 46778 \mathrm{~ms}$ & 441,66 & 81,7 & 56840,008 & 0,88 \\
\hline $31 / 10 / 2014$ & $03: 04: 46778 \mathrm{~ms}$ & 442,1 & 81,9 & 57000,008 & 0,88 \\
\hline $31 / 10 / 2014$ & $04: 04: 46778 \mathrm{~ms}$ & 449,42 & 80,8 & 56840,008 & 0,87 \\
\hline $31 / 10 / 2014$ & $05: 04: 46778 \mathrm{~ms}$ & 442,96 & 81,6 & 56813,34 & 0,88 \\
\hline $31 / 10 / 2014$ & $06: 04: 46778 \mathrm{~ms}$ & 444,28 & 81,7 & 56826,672 & 0,88 \\
\hline $31 / 10 / 2014$ & $07: 04: 46778 \mathrm{~ms}$ & 443,64 & 81,3 & 56706,672 & 0,87 \\
\hline $31 / 10 / 2014$ & $08: 04: 46778 \mathrm{~ms}$ & 438,54 & 82,9 & 56813,34 & 0,88 \\
\hline $31 / 10 / 2014$ & $09: 04: 46778 \mathrm{~ms}$ & 435,42 & 82,7 & 56640,008 & 0,88 \\
\hline
\end{tabular}




\section{RESULTADOS E DISCUSSÃO}

Em seguida serão apresentados os resultados financeiros calculados analisando a troca de 1 motor padrão por um motor de alto rendimento Premium acionado por um Inversor de frequência interligado ao controlador de temperatura.

Tabela 4. Resultados financeiros calculados
\begin{tabular}{|l|c|c|}
\hline Resultados & Motor Standard & $\begin{array}{c}\text { Motor W 22 Premium + } \\
\text { inversor de fre quência + } \\
\text { controlador de tem peratura }\end{array}$ \\
\hline Consumo anual ( $\mathrm{kWh} / \mathrm{ano}$ ) & $536.462,40$ & $239.410,79$ \\
\hline Consumo anual (R\$) & $\mathrm{R} \$ 80.469,36$ & $\mathrm{R} \$ 35.911,62$ \\
\hline Economia (\%) & \multicolumn{2}{|c|}{$\mathrm{R} \$ 44.557,74$} \\
\hline Economia (R\$) & 0,8 \\
\hline ROI(anos) & \multicolumn{2}{|c|}{0} \\
\hline
\end{tabular}

\section{Outros ganhos}

Redução no consumo de energia de aproximadamente $55 \%$

Retorno garantido sobre o capital investido

Baixo custo operacional

Variação da velocidade dos ventiladores de acordo com a necessidade do processo

Automatização do sistema

Vida útil estendida

Manutenção reduzida

Excelente relação custo-benefício

Baixos níveis de vibração e ruído

Fácil manutenção e instalação

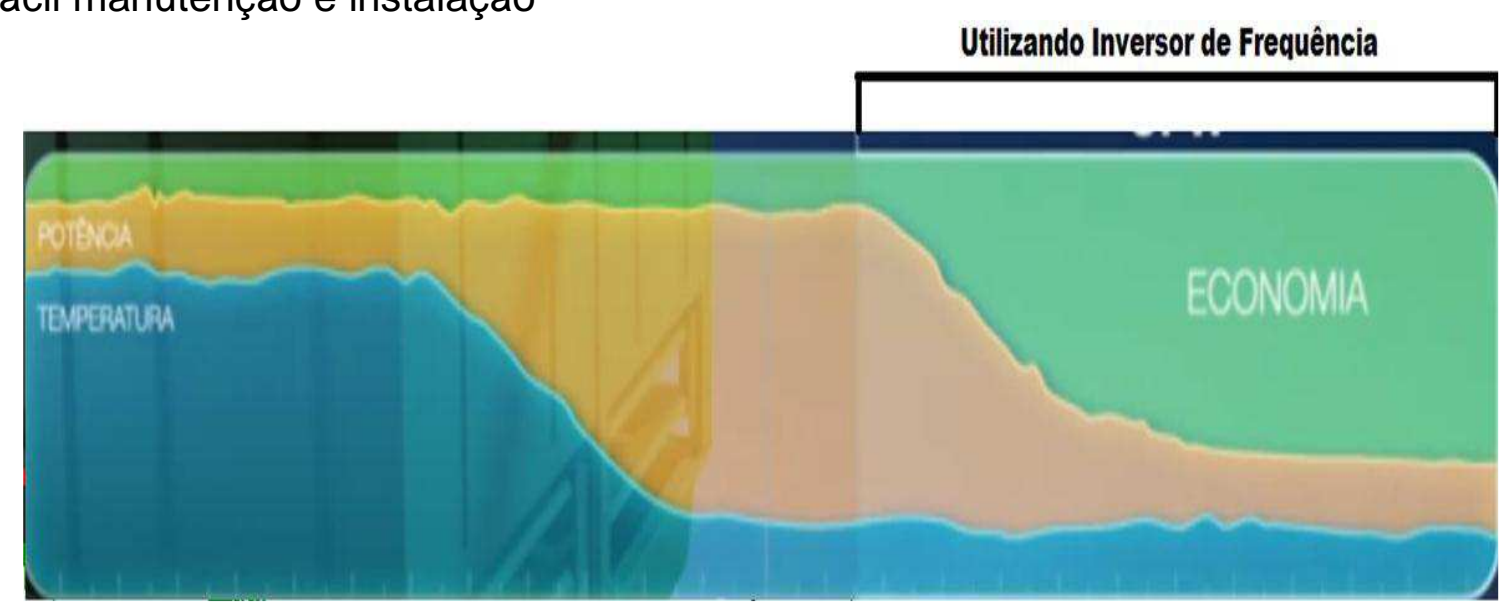

\section{CONCLUSÃO}

Fig. 2. Exemplo de Torres de Resfriamento [4].

Nas Torres de Resfriamento, ao implantar uma solução que pode a variar a velocidade dos ventiladores de acordo com a temperatura ambiente e a exigência de resfriamento do processo, pode-se obter grandes ganhos em economia de energia. No monitoramento realizado e no estudo de viabilidade econômica foi possível analisar o consumo elétrico durante um período. Desta forma, comprovamos nas medições que a aplicação da solução proporcionou uma redução de $55 \%$ no consumo de energia elétrica, obtendo assim, um retorno em 09 Meses do investimento aplicado. 


\section{REFERÊNCIAS}

1 BEN - Balanço Energético Nacional, 2012, Secretaria de Planejamento e Desenvolvimento Energético - SPE / Ministério de Minas e Energia - MME, Distrito Federal, Brasil.

2 MMA - MINISTÉRIO DO MEIO AMBIENTE. Eficiência Energética e Conservação de Energia. Disponível em: http://www.mma.gov.br/clima/energia/eficiencia-energetica. Acesso em 30 de Outubro de 2014.

3 ENSSLIN, S. R. Manuais Elektro de Eficiência Energética. Revista Contemporânea de Contabilidade, v. 7, n. 14, 2011.

4 WEG, Solução de Eficiência Energética em Torres de Resfriamento. Disponível em: http://ecatalog.weg.net/files/wegnet/WEG-solucao-de-eficiencia-energetica-em-torresde-resfriamento-estudo-de-caso-portugues-br.pdf . Acesso em 27 de Setembro de 2014. 